\title{
Modelo de bloque deslizante para analizar el comportamiento cinemático de deslizamientos en suelos a partir de las fuerzas resultantes de un modelo de equilibrio límite
}

\author{
A sliding block model to analyse the kinematic behaviour of soil landslides from the resultant \\ forces of a limit equilibrium analysis
}

Fecha de entrega: 23 de julio 2019

Fecha de aceptación: 14 de abril 2020

\section{Andrés Felipe Prieto, Laura Camila Cantor y Carlos Eduardo Rodríguez}

Departamento de Ingeniería Civil, Pontificia Universidad Javeriana, Carrera 7 No 40-62, Bogotá D.C, Colombia, aprietoc@javeriana.edu.co, cantor.1@javeriana.edu.co, crodriguezp@javeriana.edu.co

En este trabajo se plantea un modelo bidimensional de bloque deslizante -discretizado en dovelas-, en el cual se acoplan las variables geométricas, mecánicas y reológicas del deslizamiento que permite el análisis del comportamiento cinemático de los deslizamientos en suelos. El movimiento de la masa deslizada es modelado mediante un sistema de bloques adyacentes conectados mediante resortes. La resistencia al movimiento es dada por el modelo reológico Coulomb-viscoso, en el cual se consideran las componentes friccionantes y viscosas, representadas con un sistema en paralelo compuesto por un deslizador y un amortiguador, respectivamente. La ecuación de movimiento del modelo corresponde a una ecuación diferencial lineal de segundo orden. Esta es resuelta por el método de diferencias finitas, obteniendo una ecuación explicita para conocer el desplazamiento, velocidad y aceleración de cada dovela. Se realizó un algoritmo en el software MATLAB ${ }^{\circledR}$ en el cual se programaron las ecuaciones necesarias para obtener resultados de la cinemática del deslizamiento. Por medio del algoritmo, fue posible realizar un análisis de sensibilidad a los parámetros del modelo. Este fue ejecutado en el problema del bloque deslizante en el talud infinito, donde se logra conocer la influencia de cada parámetro del modelo. El modelo es aplicado al deslizamiento de Hiegaesi, ocurrido en Japón en 1998, donde se logró modelar el comportamiento cinemático teniendo en cuenta los registros reportados. Sin embargo, se considera necesario más investigación para poder aplicar el modelo propuesto en la predicción del comportamiento cinemático de deslizamientos, debido a las limitaciones discutidas en este documento.

Palabras clave: distancia de viaje, velocidad deldeslizamiento, bloque deslizante, cinemática de deslizamientos, viscosidad, ecuación de movimiento, método de diferencias finitas
In this paper, a two-dimensional sliding block model -discretized in slices- is presented, in which the geometrical, mechanical and rheological variables of the landslide are considered in order to analyse the soil landslide kinematic behaviour. The movement of the sliding mass is presented as a system of adjacent blocks; the movement strength is given by the Coulombviscous rheological model, where the friction and viscous components are considered, represented by a parallel system of a slider and a damper, respectively. The movement equation of the model corresponds to a second order differential equation, which was solved by the finite difference method, obtaining an explicit equation to know the displacement, velocity and acceleration of each block. An algorithm was developed in MATLAB ${ }^{\circledR}$ software, where movement equations were programmed to obtain results of the sliding kinematics. With the algorithm developed it was possible to make a sensitivity analysis to the parameters of the model. This was implemented in the problem of the sliding block at infinity slope, where it was possible to know the influence of each of the parameters of the model. The model has also been applied to the Hiegaesi landslide occurred in Japan in 1998, where the kinematic behaviour has been analysed in the model, regarding the records reported. However, further research is needed in order to apply the published model for prediction of the kinematic behaviour of landslides due to different limitations discussed in this paper.

Keywords: landslide runout, landslide velocity, sliding block, landslide kinematics, viscosity, movement equation, finite difference method 


\section{Introducción}

Los deslizamientos son eventos naturales que pueden llegar a afectar el tránsito de una vía, causar la destrucción de viviendas e incluso pérdidas de vidas humanas; ya que la masa deslizada logra recorrer la distancia y alcanzar la velocidad necesaria para causar afectaciones. En Colombia, DESINVENTAR (online.desinventar.org) tiene registro de más de diez mil deslizamientos que han ocurrido en los diferentes departamentos del país, y que han causado aproximadamente siete mil muertes entre 1921 y 2017. Estos eventos corresponden a los que más han generado pérdidas de vida humana entre 1970 y 2011 con un 36\%, y a su vez es el tercer evento que más ha causado viviendas destruidas con $10 \%$, respecto a los eventos estudiados por el Banco Mundial (2012) como se muestra en la Figura 1.
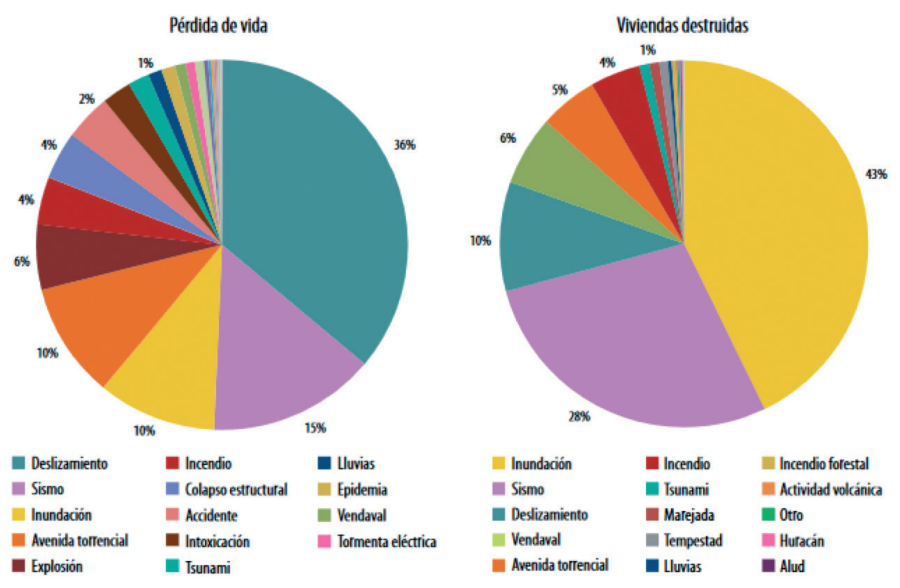

Figura 1: Pérdidas de vidas humanas y viviendas destruidas por tipo de evento entre 1970 y 2011 en Colombia (Banco Mundial, 2012)

A nivel mundial, son miles de muertes cada año causadas por los deslizamientos (Petley, 2012). Gran parte de estas muertes ocurren en zonas que topográficamente son relativamente planas, pero aun así, hacen parte de la distancia de viaje del deslizamiento. Es común que la población humana se concentre en estos lugares, donde puede ocurrir el impacto de un deslizamiento a altas velocidades, muchas veces sin advertencia alguna (McDougall, 2017). El deslizamiento ocurrido en Oso, Washington, en marzo de 2014, el cual viajó más de $1 \mathrm{~km}$ a lo largo del valle y causó 43 muertes de la comunidad de Steelhead Haven, es uno de los muchos ejemplos existentes alrededor del mundo de esta problemática (Keaton et al., 2014).

Dado que la estabilización de un talud no es siempre factible, se necesita de herramientas y métodos para predecir y analizar el comportamiento de la cinemática del deslizamiento, con el fin de definir el uso del suelo o diseñar protección en la zona de viaje y depósito del deslizamiento.

Los métodos numéricos que han sido desarrollados para analizar dichos parámetros cinemáticos pueden ser agrupados en dos categorías (McDougall, 2017). La primera corresponde a modelos empíricos-estadísticos, los cuales son desarrollados con referencia en datos de deslizamientos reales basados en la clasificación del tipo de movimiento, sin embargo, sus estudiosy correlaciones serealizan en materiales y casos específicos. La segunda categoría corresponde a métodos analíticos basados en modelación (Hunter y Fell, 2003; Glastonbury et al., 2002), que según Miao et al. (2001) se presentan en modelos a escala física -los cuales han presentado dificultades debido a efectos geométricosy modelos dinámicos, que son subdivididos en modelos de masa agrupados, modelos basados en la teoría de colisiones y modelos basados en mecánica del medio continuo. Sin embargo, cada uno de estos presenta limitaciones, como no pueden simular el movimiento de la pata del talud (Hungr, 1995), consideran únicamente resistencias friccionantes que no disipan la energía cinética durante el proceso dando lugar a grandes desplazamientos (Angeli et al., 1996) o han sido aplicados únicamente para deslizamientos tipo flujo (Chen y Lee, 2000; Crosta et al., 2003; Cuomo, 2014; Hungr, 2009).

Miao et al. (2001) presentan un modelo de bloque deslizante, el cual considera la no homogeneidad de la velocidad y deformación de la masa deslizada, simultáneamente con la energía de deformación y disipación de energía durante el deslizamiento. Sin embargo, debido a que este modelo no considera los parámetros reológicos del suelo es posible encontrar valores de distancia de viaje y velocidad de deslizamiento de magnitudes muy elevadas a diferencia de las registradas en campo (Angeli et al., 1996).

En este documento se propone un modelo para analizar el comportamiento cinemático de los deslizamientos en suelos, añadiendo una componente de resistencia viscosa al modelo de bloque deslizante propuesto por Miao et al. (2001). La aplicabilidad del modelo es verificada con el problema del talud infinito y con el deslizamiento de Hiegaesi, ocurrido en Japón en 1998. 
El método del bloque deslizante ha sido usado para estimar distancias de viaje y velocidad de movimientos en masa. Consiste en que la masa del deslizamiento se representa como bloques que se desplazan por una superficie debido a las fuerzas gravitacionales, considerando una resistencia en la base. Miao et al. (2001) desarrollaron el modelo de bloque deslizante para la predicción de deslizamientos de altas velocidades. La teoría se basa únicamente en el mecanismo de la distancia de viaje y las altas velocidades de los deslizamientos. La rápida y gran caída del centro de gravedad de la parte trasera del deslizamiento es considerada la fuente dinámica y la alta velocidad es debida a la disminución resultante de la fuerza de fricción. El modelo fue desarrollado bajo la suposición que los bloques se mueven continuamente, esto es, ellos no se separan a nivel de macro escala, mientras la altura y el ancho de cada bloque varían durante el transporte (Miao et al., 2001). Se considera como fuerza resistente una componente friccionante uniforme; sin embargo, los efectos viscosos del suelo no se consideran.

En el modelo propuesto en este trabajo, se realizaron diferentes modificaciones con el fin de considerar efectos viscoplásticos en los deslizamientos. A continuación, se presenta el desarrollo metodológico llevado a cabo para el planteamiento del modelo.

\section{Fuerzas actuantes y resistentes en un talud}

Así como en los modelos de equilibrio límite clásicos, en esta metodología el deslizamiento es considerado como un problema de deformación plana discretizado mediante dovelas; además se asume que la superficie de falla crítica es conocida. Las fuerzas actuantes y resistentes para cada dovela, se muestran en la Figura 2. Estas corresponden a las asumidas en el método Unbalanced Thrust Method UTM (GB50021, 1995), donde las fuerzas entre dovelas actúan en la altura media de la dovela y en dirección paralela a la superficie de falla. Para la dovela $i, W_{\mathrm{i}}$ corresponde al peso, $P_{\mathrm{i}}$ es la fuerza actuando entre las dovelas, $N_{\mathrm{i}}$ es la fuerza normal en la base de la dovela, $T_{\mathrm{i}}$ es la fuerza resistente que se opone al movimiento y $U_{\mathrm{i}}$ es la fuerza ejercida por la presión de poros que actúa en la base de las dovelas. Adicionalmente, $\alpha_{\mathrm{i}}, b_{\mathrm{i}}$ y $h_{\mathrm{i}}$ corresponden respectivamente al ángulo de inclinación en la base de la dovela, el ancho de la dovela y su altura respecto al punto medio de las tajadas en análisis.

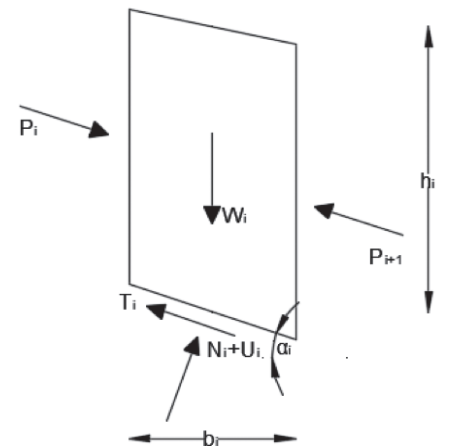

Figura 2: Diagrama de fuerzas actuantes en la dovela en el método UTM

Para aplicar el modelo propuesto, es válido usar los diferentes métodos de equilibrio límite en los cuales el talud es discretizado en dovelas y entre ellas actúa una fuerza. Sin embargo, en caso de implementar otro modelo de equilibrio límite (diferente a UTM), se deben determinar fuerzas equivalentes a las supuestas en esta metodología.

Se trabajarán deslizamientos en suelos clasificados de rápidos a extremadamente rápidos, según la clasificación de Hungr et al. (2014). El deslizamiento se modeló como un problema de deformación plana, en el cual no se considera la causa del deslizamiento; el modelo se enfoca principalmente en el mecanismo de viaje de la masa deslizada. Este modelo parte de la condición inicial de las fuerzas entre dovelas que actúan al momento que ocurre la falla, obtenidas a partir de un método de equilibrio límite. Durante el movimiento, el ancho y la altura de cada dovela varían manteniendo su volumen constante. Adicionalmente, la acumulación y liberación de la energía de deformación ocurre durante el transporte y es tenida en cuenta mediante resortes que conectan las dovelas. La masa deslizada se divide en $n$ bloques, contados secuencialmente desde la corona hasta el pie del deslizamiento; en cada bloque actúan las fuerzas presentadas previamente. Así como las fuerzas entre dovelas, la aceleración se considera paralela a la superficie de falla. El modelo fue planteado en términos de esfuerzos efectivos. Durante el movimiento de la masa deslizada, la presión de poros actúa en la base de cada dovela como una fuerza desestabilizante y el nivel freático se considera proporcional a la altura de cada dovela respecto a su posición inicial. El movimiento es simplificado a un modelo de bloques conectados por resortes, y en la base de cada dovela se consideran las componentes de resistencia friccionante y viscosa, representadas mediante un sistema en paralelo de un slider y un amortiguador. 


\section{Modelo de masa conectado por resortes}

En la Figura 3 se esquematiza el modelo simplificado de masa conectado por resortes.

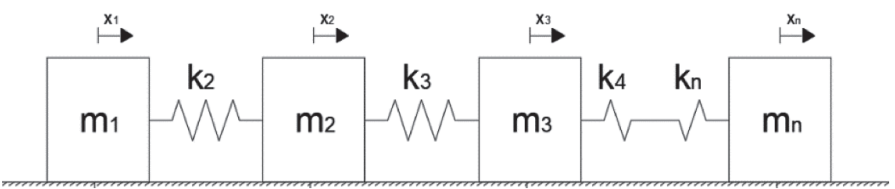

Figura 3: Diagrama de masas conectadas por resortes (Miao et al., 2001)

Los resortes representan un comportamiento elástico entre las dovelas durante el movimiento, y se relacionan con la acumulación y liberación de la energía de deformación. La fuerza horizontal ejercida sobre el bloque $i$ por el bloque $i$ - 1 , es obtenida a partir de la ley de Hooke:

$$
F_{i}=k_{i} \delta_{i}
$$

donde $\delta_{i}$ es la variación del ancho de la tajada (positiva en compresión) y $k_{i}$ es la constante de rigidez del resorte. Para determinar la constante de rigidez de los resortes, se adoptó lo propuesto por Miao et al. (2001) y Mora (2011), donde al suponer la dovela como un rectángulo e igualar la energía de deformación axial con la energía potencial elástica, se expresa $k_{i}$ en función del módulo de elasticidad del material y de la sección de las tajadas.

$$
k_{i}=E_{0} \frac{h_{0 i}}{b_{O i}}
$$

Se parte de la premisa que el módulo de elasticidad seleccionado depende del nivel de deformación al cual se presente el movimiento del deslizamiento. Sin embargo, conocer estas tasas de deformación en los deslizamientos es un área de estudio que necesita mayor investigación. Por esto, la selección de este parámetro será gobernada en esta investigación por el análisis de sensibilidad y retro cálculo. Consecuentemente, es una de las limitaciones por las cuales no se podrá hacer uso de este modelo en predicciones, sin haber realizado investigación más exhaustiva en este aspecto. Más adelante se volverá a discutir al respecto.

\section{Modelo reológico Coulomb viscoso}

Los modelos para determinar la distancia de viaje de deslizamientos que consideran únicamente componentes de resistencia friccionante, dan lugar a grandes desplazamientos y velocidades, a diferencia de las registradas en campo (Angeli et al., 1996). Además, se ha evidenciado la existencia de fuerzas viscosas en los deslizamientos, donde la componente viscosa, acoplada con la ecuación de movimiento, ha llevado a resultados precisos (Corominas et al., 2005). A partir de observaciones de campo y experimentos en flujos de detritos, Johnson (1970) ha propuesto que la resistencia dinámica total es una combinación del esfuerzo de fluencia y de la resistencia friccional y viscosa. Johnson (1970) realizó una modificación al esfuerzo de fluencia del modelo de Bingham, dividiendo este en las componentes de cohesión y fricción, dando lugar al modelo Coulomb viscoso (Chen y Lee, 2002). Teniendo en cuenta la suposición en la que durante el movimiento del deslizamiento no actúan fuerzas cohesivas $\left(\tau_{c}=0\right)$, se tuvo en cuenta únicamente la componente de resistencia friccional y viscosa, mediante un sistema en paralelo de un slider y un amortiguador, respectivamente (Figura 4); tal como lo propuso Angeli et al. (1996).

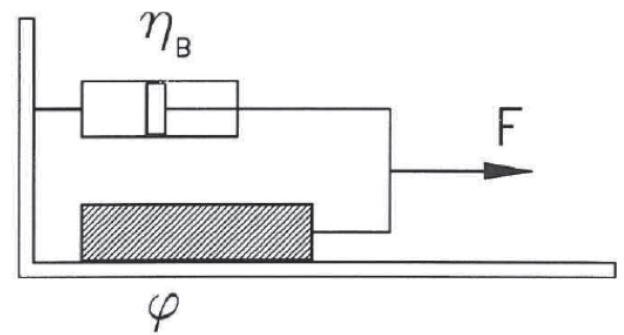

Figura 4: Modelo reológico (Angeli et al., 1996)

Este modelo Coulomb viscoso es considerado como viscoplástico (Wang, 2008), en el cual se encuentra la ecuación friccional de Coulomb $T_{i}=N_{i} \tan \varphi_{d i}$ y un término en función del coeficiente de viscosidad. Esta componente reológica actuará en dirección opuesta al movimiento de los bloques, actuando como una fuerza resistente. En este caso, el término $T_{i}$ corresponde a:

$$
T_{i}=N_{i} \tan \varphi_{i}^{\prime}+\mu_{i} \frac{d x_{i}}{d t}
$$

donde $\varphi_{i}^{\prime}$ es el ángulo de fricción dinámica entre el suelo deslizado y la superficie recorrida, $\mu$ es el coeficiente de viscosidad dinámica y $\frac{d x}{d t}$ es la velocidad de la masa. A partir de las suposiciones realizadas y de los modelos planteados, se tiene un modelo de masa conectado por resortes, con una fuerza resistente correspondiente al modelo reológico Coulomb viscoso; esquematizado en la Figura 5. 


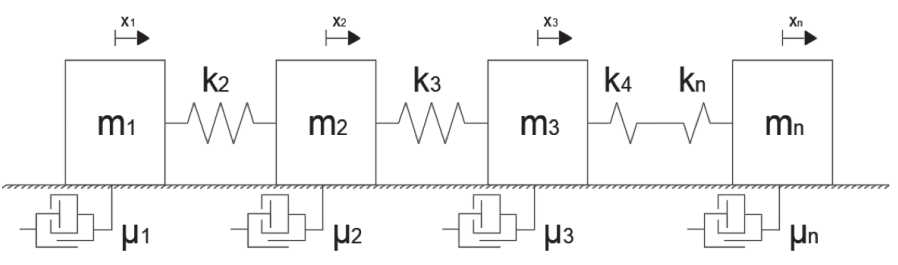

Figura 5: Diagrama de masa conectado por resortes y amortiguadores

Para determinar la ecuación de movimiento, se realizó el equilibrio de fuerzas para cada dovela en dirección paralela y perpendicular a la superficie de falla, considerando la segunda ley de Newton. Las ecuaciones de movimiento se desarrollaron para la primera dovela, dovelas intermedias y para la última dovela. En la Figura 6 se pueden observar las fuerzas actuantes en la dovela intermedia del modelo propuesto.

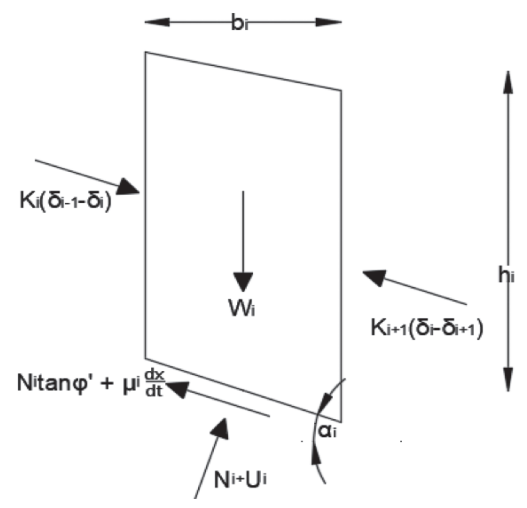

Figura 6: Diagrama de fuerzas en una dovela intermedia

Las fórmulas (8) y (9) se obtuvieron en función de las siguientes constantes:

$$
\begin{aligned}
& A_{i}=k_{i}\left[\cos \left(\alpha_{i-1}-\alpha_{i}\right)-\operatorname{sen}\left(\alpha_{i-1}-\alpha_{i}\right) \tan \left(\varphi^{\prime}\right)\right] \\
& C=\frac{\mu_{i}}{\Delta t} \\
& D=\frac{m_{i}}{\Delta t^{2}} \\
& E_{i}=W_{i}\left[\operatorname{sen} \alpha_{i}-\cos \alpha_{i} \tan \varphi^{\prime}\right]+U_{i} \tan \varphi^{\prime}
\end{aligned}
$$

Inicialmente se realizó sumatoria de fuerzas en dirección $\hat{\boldsymbol{\imath}}$ (paralelo a la superficie de falla) y en dirección $\hat{\boldsymbol{J}}$ (perpendicular a la superficie de falla).

$\Sigma F_{j}=N_{i}-W_{i} \cos \alpha_{i}-k_{i}\left(\delta_{i-1}-\delta_{i}\right) \operatorname{sen}\left(\alpha_{i-1}-\alpha_{i}\right)+U_{i} L_{i}=0$

$\Sigma F_{i}=W_{i} \operatorname{sen} \alpha_{i}+k_{i}\left(\delta_{i-1}-\delta_{i}\right) \cos \left(\alpha_{i-1}-\alpha_{i}\right)-k_{i+1}\left(\delta_{i}-\delta_{1+1}\right)-\cdots$
Despejando $N$ se obtiene:

$N_{i}=W_{i} \cos \alpha_{i}+k_{i}\left(\delta_{i-1}-\delta_{i}\right) \operatorname{sen}\left(\alpha_{i-1}-\alpha_{i}\right)-U_{i} L_{i}$

Reemplazando (10) en (9) se obtiene:

$$
\begin{aligned}
& m_{i} \alpha_{i}+\mu_{i} v_{i}=W_{i} \operatorname{sen} \alpha_{i}-W_{i} \cos \alpha_{i} \tan \varphi^{\prime}-k_{i+1}\left(\delta_{i}-\delta_{i+1}\right)+\cdots \\
& \ldots k_{i}\left[\left(\delta_{i-1}-\delta_{i}\right) \cos \left(\alpha_{i-1}-\alpha_{i}\right)-\left(\delta_{i}-\delta_{i+1}\right) \operatorname{sen}\left(\alpha_{i-1}-\alpha_{i}\right) \tan \varphi^{\prime}\right]
\end{aligned}
$$

Finalmente,

$$
0=E_{i}-k_{i+1}\left(\delta_{i}-\delta_{i+1}\right)+\left(\delta_{i-1}-\delta_{i}\right)\left[A_{i}\right]-m_{i} \frac{\partial^{2} x}{\partial t^{2}}-\mu_{i} \frac{\partial x}{\partial t}
$$

Donde

$\delta_{i}=x_{i, t}-x_{i, t o}$

Y $x_{i, t 0}$ corresponde a la posición inicial de la dovela $i$. El procedimiento anteriormente mencionado, se siguió para la primera y última dovela (condiciones de frontera); y en seguida, se pueden observar los diagramas de fuerzas en cada caso.
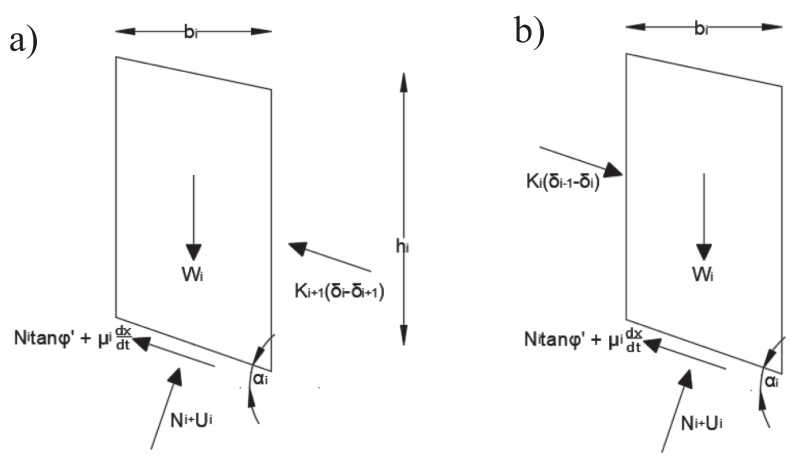

Figura 7: a) Diagrama de fuerzas en la primera dovela y b) última dovela

Las ecuaciones planteadas para la primera dovela (corona del deslizamiento) y última dovela (pata del deslizamiento), son presentadas en las fórmulas (14) y (15), respectivamente.

$$
\begin{aligned}
& 0=E_{i}-k_{i+1}\left(\delta_{i}-\delta_{i+1}\right)-m_{i} \frac{\partial^{2} x_{i}}{\partial t^{2}} \\
& 0=E_{i}+\left(\delta_{i-1}-\delta_{i}\right)\left[A_{i}\right]-m_{i} \frac{\partial^{2} x_{i}}{\partial t^{2}}-\mu \frac{\partial x_{i}}{\partial t}
\end{aligned}
$$

El modelo propuesto posee limitaciones. Al ser un modelo bidimensional (deformación plana), no tiene en cuenta la topografía tridimensional de la superficie de la trayectoria del deslizamiento, donde la masa deslizada se podría separar y cambiar de dirección durante el movimiento. No 
se tiene en cuenta la causa del deslizamiento. Se considera que la masa deslizada no cambia de volumen en el modelo, por lo cual, no es posible tener en cuenta la reacomodación de partículas que se da durante el movimiento. La interacción entre los bloques se da únicamente en las caras laterales. Los bloques tienen un solo grado de libertad, correspondiente a la dirección del movimiento. El modelo reológico seleccionado considera que la relación entre el esfuerzo cortante y la tasa de corte es directamente proporcional. Sin embargo, en suelos finos, la viscosidad decrece gradualmente con el incremento de la tasa de corte. Por tanto, en este modelo se puede sobrestimar el esfuerzo cortante real del material. La solución de la ecuación diferencial es estimada mediante diferencias finitas. La estabilidad numérica de este método es fuertemente dependiente de la discretización y de los parámetros de la ecuación. Su aplicabilidad para predicción de distancias de viaje se encuentra limitada por la selección de parámetros.

\section{Solución por diferencias finitas}

La ecuación de movimiento obtenida es una ecuación diferencial lineal de segundo orden. El método establecido en este estudio para la solución de la ecuación es el método de diferencias finitas MDF, en el cual la solución es linealmente aproximada por medio de la discretización del espacio y del tiempo. Este método tiene la ventaja de que las expresiones desarrolladas son sencillas de implementar algebraicamente en la ecuación de movimiento, y se pueden obtener resultados para cada intervalo de espacio y de tiempo.

Para solucionar la ecuación de movimiento, es necesario tener como condiciones iniciales la posición en el tiempo $t$-1 y en el tiempo $t$, para obtener numéricamente la posición de cada dovela en el tiempo $t+1$. Por esta razón, el movimiento tiene que ser analizado en dos etapas.

La primera etapa consiste en el primer movimiento, que se obtiene a partir del análisis de equilibrio límite. Los métodos considerados previamente, permiten obtener el factor de seguridad FS y las fuerzas entre las dovelas. A partir del equilibrio de fuerzas en cada dovela, se puede obtener la aceleración, velocidad y desplazamiento; considerando como condición inicial las fuerzas obtenidas al momento que ocurre la falla. Hungr (1995) considera una condición inicial de suelo en reposo, lo que desconoce las deformaciones del terreno antes de manifestarse la falla. En el modelo se asume como condición inicial las fuerzas entre las partículas que se obtienen a partir de análisis de equilibrio límite. Las fuerzas iniciales entre las tajadas se toman para la condición en la que el FS $=1.0$. En el modelo se trabajó como condición crítica $|F S-1| \leq$ 0.05 , para tener en cuenta las incertidumbres en el método de cálculo. La obtención de las variables cinemáticas para el primer movimiento, siguiendo el método UTM y el correspondiente procedimiento algebraico, se basa en lo descrito por Miao et al. (2001). Finalmente, las coordenadas obtenidas en función de las fuerzas entre dovelas en su condición de falla son el parámetro de entrada para el cálculo de la segunda etapa del deslizamiento.

La segunda etapa consiste en el movimiento de la masa deslizada por la superficie una vez ocurra el primer movimiento. Con base en lo que se ha mencionado anteriormente, y en el modelo que se planteó para el cálculo de las variables cinemáticas de los deslizamientos, se desarrolló la solución de la ecuación de movimiento con el fin de obtener las expresiones para obtener la distancia de viaje y velocidad de deslizamientos. Haciendo uso de la fórmula (16), se procedió a despejar explícitamente la variable de la posición en la ecuación diferencial, aplicando la solución por MDF. El procedimiento algebraico se realizó con el fin de obtener las expresiones para las tres condiciones de las dovelas en las que se discretiza el deslizamiento. A continuación, se puede observar desde la fórmula (16) a la (18), la solución de la ecuación para la primera, intermedia y última dovela, respectivamente.

En los casos en que el valor obtenido de $x_{\mathrm{i}, t+1}$ es menor a

$$
\begin{aligned}
& x_{i, t+1}=\frac{E_{i}+x_{i+1, t_{0}}\left[k_{i+1}\right]+x_{i, t}\left[C_{i}+2 D_{i}-k_{i+1}\right]+x_{i+1, t}\left[k_{i+1}\right]+x_{i, t_{0}}\left[k_{i+1}\right]+x_{i, t-1}\left[-D_{i}\right]}{C_{i}+D_{i}} \\
& =\frac{x_{i, t+1}+x_{i-1, t_{0}}\left[-A_{i}\right]+x_{i+1, t_{0}}\left[k_{i+1}\right]+x_{i-1, t}\left[A_{i}\right]+x_{i, t}\left[C_{i}+2 D_{i}-A_{i}-k_{i+1}\right]+x_{i+1, t}\left[k_{i+1}\right]+x_{i, t_{0}}\left[A_{i}+k_{i+1}\right]+x_{i, t-1}\left[-D_{i}\right]}{\left[C_{i}+D_{i}\right]} \\
& x_{i, t+1}=\frac{E_{i}+x_{i-1, t_{0}}\left[-A_{i}\right]+x_{i+1, t_{0}}\left[k_{i+1}\right]+x_{i-1, t}\left[A_{i}\right]+x_{i, t}\left[C_{i}+2 D_{i}-A_{i}\right]+x_{i, t_{0}}\left[A_{i}\right]+x_{i, t-1}\left[-D_{i}\right]}{C_{i}+D_{i}}
\end{aligned}
$$


$x_{\mathrm{i}, t}$, indican que el bloque $i$ estaría retrocediendo, lo cual no es consecuente con el movimiento de un deslizamiento. En los casos en que ocurra esto, se considera que el bloque mantiene su posición, es decir:

$$
\text { Si } x_{i, t+1}<x_{i, t} \text { entonces } x_{i, t+1}=x_{i, t}
$$

\section{Algoritmo LAAND - Slide Runout}

La ecuación obtenida, a pesar de ser una ecuación explícita, tiene un alto grado de complejidad debido a la cantidad de parámetros que intervienen en la solución. Con el fin de optimizar el tiempo que conlleva obtener un resultado para cada dovela y en cada intervalo de tiempo, fue necesario acudir a la programación. El algoritmo, desarrollado en el software Matlab ${ }^{\circledR}$, permite determinar la distancia de viaje y velocidad de los deslizamientos a partir de la solución de las ecuaciones desarrolladas. El programa desarrollado, denominado LAAND - Slide Runout-, se divide en varios módulos y funciones que componen el diagrama de flujo del algoritmo desarrollado, presentado en la Figura 8. Como salida del programa, se obtienen los desplazamientos de cada dovela en los intervalos de tiempo determinados, así como la velocidad y la aceleración.

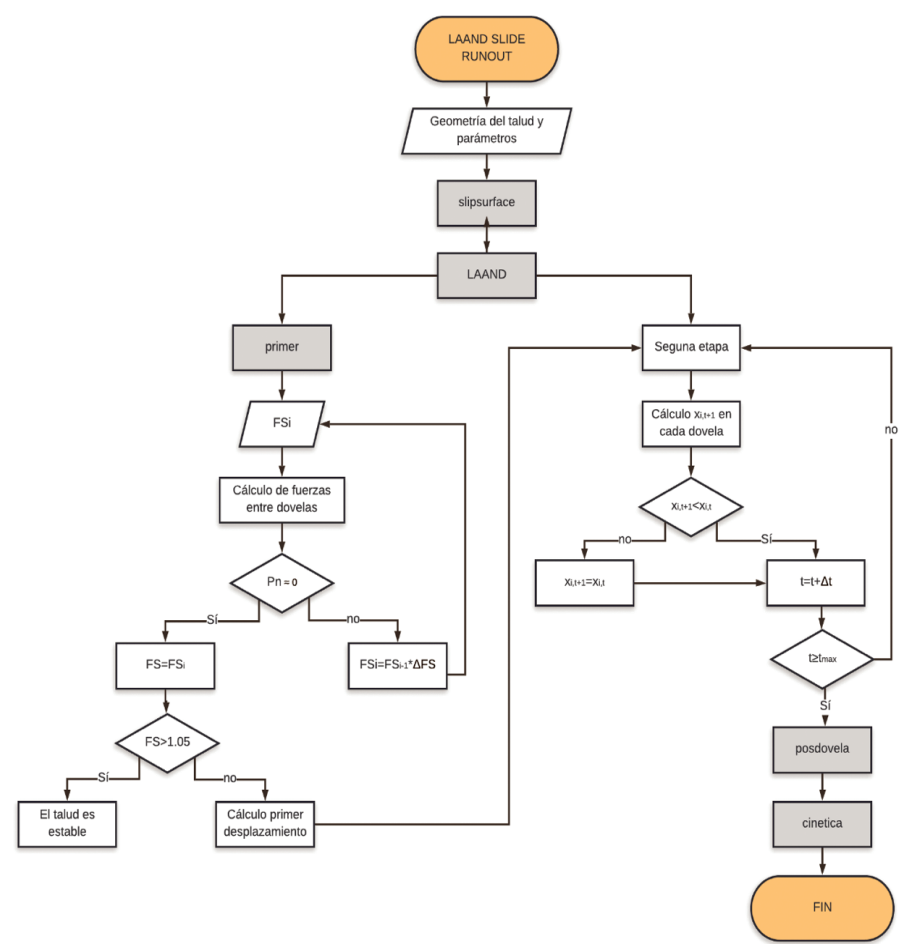

Figura 8: Diagrama de flujo de algoritmo LAAND-Slide Runout
Análisis de sensibilidad de parámetros en el problema del talud infinito

Para analizar la sensibilidad que tienen los parámetros de la ecuación de movimiento, se aplicó el modelo propuesto al problema del bloque deslizante en un talud infinito. En este caso, consiste en un bloque rígido supuesto de $70 \mathrm{~m}$ de ancho y $50 \mathrm{~m}$ de altura que se desliza por una superficie infinita con una inclinación de $26^{\circ}$. El planteamiento de este problema permite normalizar la pendiente y el área de las dovelas, al considerar una superficie del deslizamiento constante e infinita, y dovelas que tienen sus dimensiones iguales al discretizar la masa deslizada. El análisis de sensibilidad se realizó con los parámetros $E$ (módulo de deformación), $\mu$ (coeficiente de viscosidad dinámico), $\Delta t$ (intervalo de tiempo), número de dovelas y $\varphi$ ' (ángulo de fricción dinámico). Para esto, se establecieron parámetros constantes y se varió cada uno de estos independientemente en diferentes rangos (ver Tabla 1).

Tabla 1: Valores seleccionados de los parámetros del modelo

\begin{tabular}{|l|c|c|c|}
\hline Parámetro & Magnitud & Variación & Rango n \\
\hline $\begin{array}{l}\text { Módulo de deformación } \\
E, \mathrm{kPa}\end{array}$ & 100 & $10^{\mathrm{n}}$ & {$[-1,6]$} \\
\hline $\begin{array}{l}\text { Coeficiente } \\
\text { viscosidad } \mu, \mathrm{kPa}-\mathrm{s}\end{array}$ & 50 & $10^{\mathrm{n}}$ & {$[-1,6]$} \\
\hline Intervalo de tiempo $\Delta t, \mathrm{~s}$ & 0.1 & $10^{\mathrm{n}}$ & {$[-2,1]$} \\
\hline Número de dovelas & 10 & $\mathrm{~N}$ & {$[2,30]$} \\
\hline $\begin{array}{l}\text { Ángulo de fricción } \\
\text { dinámico } \varphi^{\prime},{ }^{\circ}\end{array}$ & 15 & $\mathrm{~N}$ & {$[4,30]$} \\
\hline
\end{tabular}

Como resultado, se obtuvieron las gráficas de posición, velocidad y aceleración en el tiempo como se muestra en la Figura 9.

Los análisis realizados para cada uno de los parámetros son presentados en la Figura 10. Debido a que, para cierta combinación de parámetros, se obtienen resultados en los que la posición de ciertas dovelas tiende al infinito y no reflejan el comportamiento esperado del movimiento de la masa deslizada, el promedio de la distancia de viaje puede dar tanto un número muy grande $\left(>10^{5} \mathrm{~m}\right)$, o simplemente coincidir con un rango de distancia de viaje considerado como plausible, i.e. el caso en que una de sus dovelas haya viajado grandes distancias a comparación de las demás. Por lo anterior, se calculó la desviación estándar DE de 

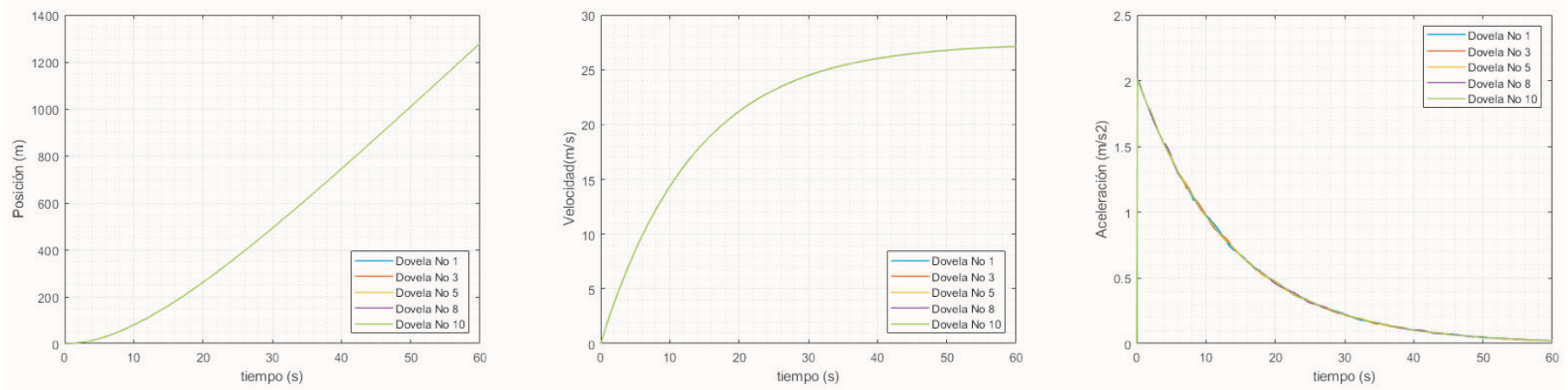

Figura 9: Posición, velocidad y aceleración del bloque deslizante.
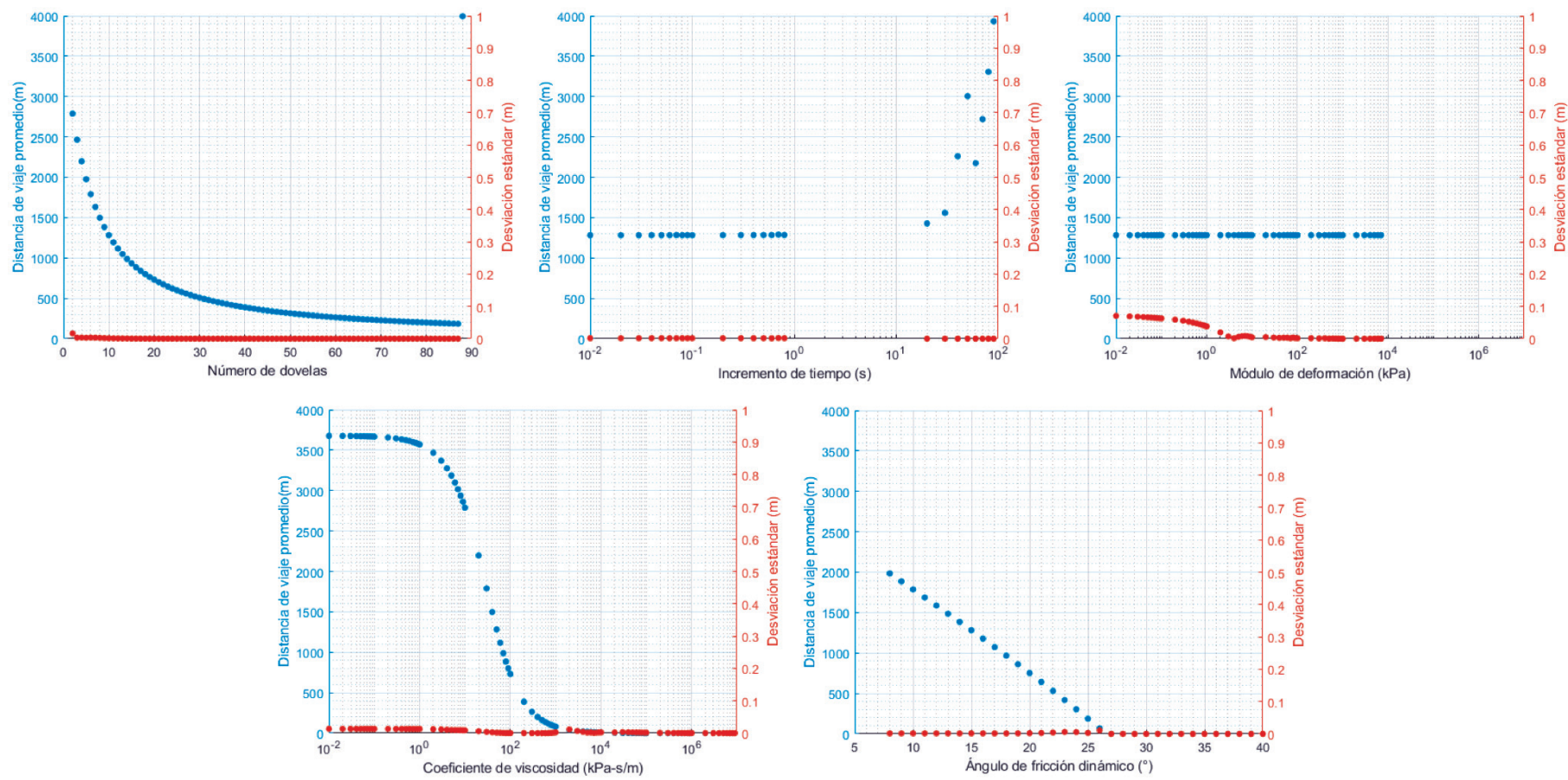

Figura 10: Análisis de sensibilidad de bloque deslizante, Modelo 2

la distancia de viaje de las dovelas, donde valores grandes de DE indican que se obtuvo resultados que conllevaron a una inestabilidad numérica. Mientras que valores cercanos a cero indican que las dovelas viajaron una distancia de viaje similar entre sí, lo cual implica que el resultado es válido. En términos generales, se observa que el módulo de deformación $E$ no tiene influencia en la distancia de viaje. Sin embargo, sí tiene influencia en la estabilidad numérica del modelo, ya que solo se obtuvieron resultados válidos para valores de $E$ que se encuentran entre 0.1 y $10000 \mathrm{kPa}$. Desde el punto de vista de la geotecnia convencional, no consideramos que este módulo esté relacionado a un módulo de elasticidad o módulo de corte. Por el contrario, y según el comportamiento numérico obtenido, corresponde a un parámetro de ajuste numérico.
Este comportamiento es justificado, ya que $E$ actúa en la interacción que hay entre las caras de las dovelas, más no es una fuerza resistente en la base de las dovelas, por lo cual, no se espera que influya en la distancia de viaje. Los valores de $E$ que no se encuentren en el rango establecido, conllevan a la inestabilidad numérica del modelo, para el ejemplo modelado y bajo los parámetros establecidos.

El coeficiente de viscosidad $\mu$ es el parámetro que más influencia tiene en la distancia de viaje. A pesar de ser un parámetro que al estimarlo varía en órdenes de magnitud (van Asch et al., 2007), tiene una relación fuertemente inversa con la distancia de viaje, es decir, que a mayor $\mu$ menor es la distancia de viaje. Así mismo, se observa que, en función de los parámetros establecidos, puede llegar a influir en la estabilidad del modelo. 
El incremento de tiempo $\Delta t$ tiene un comportamiento similar a $E$, porque no influye en la distancia de viaje, pero sí en la estabilidad numérica del modelo. Al ser un modelo de diferencias finitas, la estabilidad depende de la relación entre la discretización del tiempo, por lo cual, para los parámetros establecidos en cada modelo, será estable para $\Delta t$ inferiores de $1 \mathrm{~s}$.

El número de dovelas influye tanto en la distancia de viaje como en la estabilidad del modelo. La relación entre el número de dovelas y la distancia de viaje es de tipo exponencial negativa. Lo anterior indica que la discretización del espacio conlleva a una convergencia del modelo, donde a mayor número de dovelas, el resultado de la distancia de viaje tiende a estabilizarse para un solo valor. Sin embargo, en el análisis se observó que, con los parámetros establecidos, se puede hacer una discretización de hasta 86 dovelas; para valores superiores, el modelo es considerado como inestable numéricamente

El ángulo de fricción dinámico $\varphi$ ' influye únicamente en la distancia de viaje. El resultado indica que la distancia de viaje es inversamente proporcional al ángulo de fricción efectivo entre el material y la superficie por donde viaja la masa deslizada. Cuando el valor del ángulo de fricción es igual al ángulo de inclinación de la superficie, el bloque no se desplaza. El anterior resultado es consistente con el modelo de un talud infinito, el cual cuando el ángulo de fricción es mayor, el bloque deslizante es estable (se encuentra estático).

Adicionalmente, se realizaron gráficas en las que se presentan dos variables independientes simultáneamente, con el fin de observar la respuesta del modelo con diferentes combinaciones de parámetros. Las variables que se relacionaron fueron $E$ y $\mu$, con el fin de evidenciar si existe alguna relación entre los parámetros, bajo las cuales se obtengan resultados considerados como válidos. Además, se relacionaron el número de dovelas y $\Delta t$, debido a que la solución de la ecuación de movimiento por diferencias finitas depende de la relación entre la discretización del espacio y del tiempo. La gráfica que relaciona $E$ y $\mu$, presentada en la Figura 11, indica que para $E>10^{4} \mathrm{kPa}$, debe existir una relación proporcional con $\mu$ para obtener resultados válidos; de lo contrario, el modelo se vuelve inestable numéricamente.
En la Figura 12 se observa que para discretizar más el espacio (mayor número de dovelas), es necesario discretizar más el tiempo (menor $\Delta t$ ); lo cual es consecuente con los criterios de estabilidad para el método de diferencias finitas. En la Figura 12 es posible observar que para $\Delta t=0.1 \mathrm{~s}$ el modelo se puede discretizar como máximo hasta 86 dovelas. Al incrementar $\Delta t$ ó el número dovelas se evidencia que el modelo ya es inestable; mientras que para $\Delta t=0.01 \mathrm{~s}$, el deslizamiento se puede discretizar en 100 dovelas y el modelo numérico sigue siendo estable.

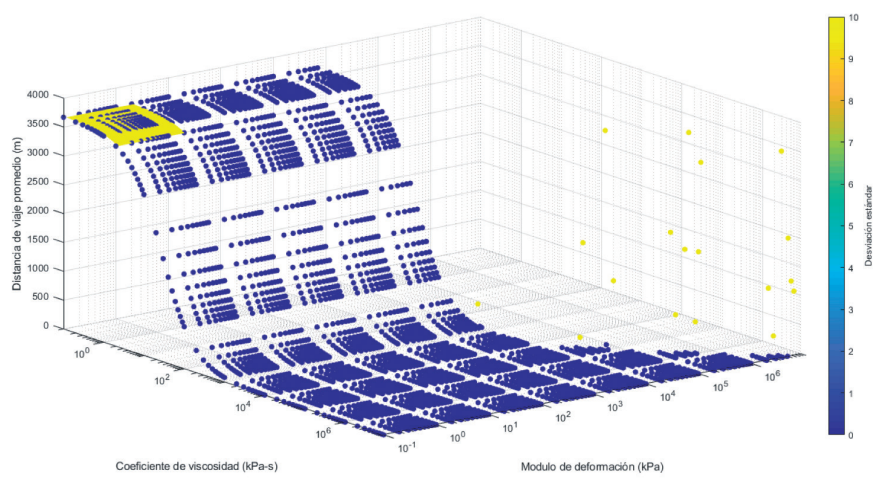

Figura 11: Análisis de sensibilidad de $E$ versus $\mu$

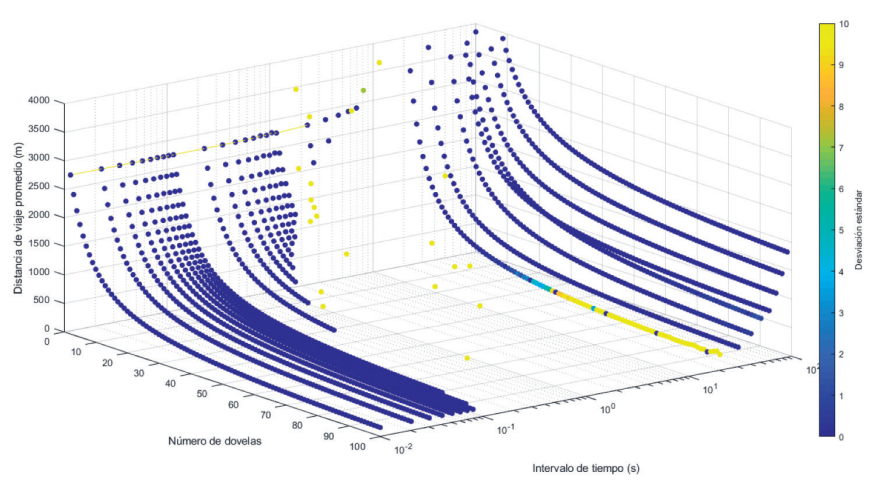

Figura 12: Análisis de sensibilidad del número de dovelas versus incremento de tiempo, Modelo 2

Se resalta que el análisis de sensibilidad realizado corresponde para el caso del bloque deslizante en el talud infinito, en el cual la inclinación de la superficie que recorre la masa deslizada se mantiene constante y el área inicial de cada una de las dovelas es la misma. La sensibilidad de los parámetros puede estar influenciada en otros casos por estas variables mencionadas, por lo cual cada caso debe ser analizado en detalle.

\section{Aplicación al deslizamiento de Hiegaesi}

El deslizamiento de Hiegaesi ocurrió al sur de Fukushima en Japón debido a las fuertes lluvias que se presentaron 
del 26 al 31 de agosto de 1998, cuya masa deslizada fue aproximadamente $1200 \mathrm{~m}^{3}$ de suelo y viajó a lo largo del valle depositándose en un cultivo de arroz. Este fue un movimiento muy superficial, que se produjo debido a la presencia de agua en sedimentos volcánicos. En los registros de la lluvia se encontró precipitación continua de 17 horas desde el 26 hasta el 27 de agosto, con un valor de 227.6 $\mathrm{mm}$, la precipitación máxima por hora fue de $90 \mathrm{~mm}$ y la precipitación acumulada antes del deslizamiento fue de 387 mm (Wang et al., 2002). La masa deslizada se encontraba lo suficientemente húmeda para afectar la estabilidad de la ladera, catalogándose entonces como un deslizamiento de suelo de larga trayectoria. En la Figura 13 se observan las distancias de viaje máximas. Se encontró que el área de origen tenía $23 \mathrm{~m}$ de ancho y $30 \mathrm{~m}$ de largo, con una altura máxima de alrededor de $3 \mathrm{~m}$ y una pendiente $25^{\circ}$.

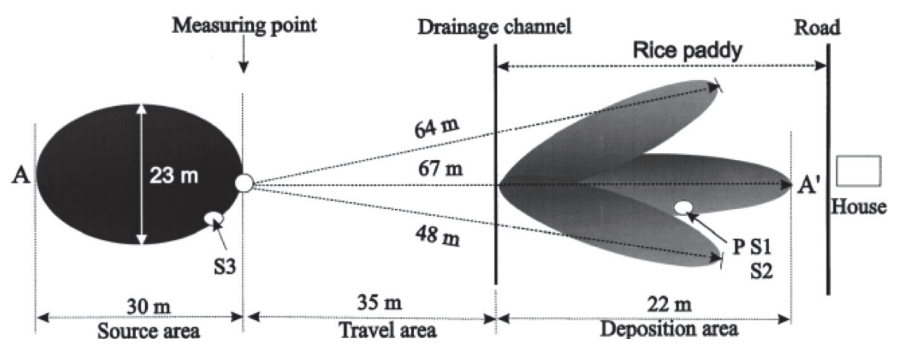

Figura 13: Secciones del deslizamiento (Wang et al., 2002)

Se reportan ensayos de corte controlado y de corte drenados y no drenados con el fin de identificar los parámetros de la masa deslizante, compuesta por una capa de ceniza volcánica franca, una capa de piedra pómez y una toba desgastada; encontrándose que el deslizamiento resultó de la licuefacción de la superficie de falla. Adicionalmente, con base en estos ensayos, fue posible determinar el ángulo de fricción residual y el ángulo de fricción máximo, $8^{\circ}$ y $41^{\circ}$, respectivamente (Wang et al., 2002).

Con el fin de validar el modelo con este caso de estudio, se definieron los parámetros en condición estática y condición dinámica. Para evaluar la estabilidad de la ladera por el método de equilibrio límite se discretizó la masa deslizada en 10 dovelas y se tuvieron en cuenta parámetros $\phi^{\prime}=40^{\circ}, \mathrm{c}^{\prime}=0 \mathrm{kPa}$ y $\gamma=16 \mathrm{kN} / \mathrm{m}^{3}$. El detonante del deslizamiento fueron las fuertes lluvias presentadas, por lo cual fue necesario tener en cuenta el nivel freático. Se consideró que el nivel freático se encontraba a una altura del $70 \%$ respecto a la altura de cada dovela, lo cual genera la inestabilidad de la ladera en el análisis de equilibrio límite. Con estos parámetros se encontró un $\mathrm{FS}=0.92$. Por otro lado, la caracterización de materiales en condiciones dinámicas es difícil de estimar debido a su complejidad para su determinación, por lo cual, los parámetros se determinaron con retro cálculo teniendo en cuenta las características geométricas del deslizamiento. Según el análisis de sensibilidad realizado previamente, se adoptaron valores de $E, \Delta t$ y número de dovelas para garantizar la estabilidad numérica, ya que estos no influyen en la distancia de viaje. A su vez, $\mu$ y $\varphi$ ' se establecieron de tal forma que se obtuviera la distancia de viaje reportada por Wang et al. (2002).

Tabla 2: Valores de los parámetros del deslizamiento Hiegaesi

\begin{tabular}{|l|c|}
\hline Parámetro & Valor \\
\hline Módulo de deformación $E, \mathrm{kPa}$ & 100 \\
\hline Coeficiente de viscosidad $\mu, \mathrm{kPa}-\mathrm{s}$ & 3 \\
\hline Intervalo de tiempo $\Delta t, \mathrm{~s}$ & 0.1 \\
\hline Número de dovelas & 10 \\
\hline Ángulo de fricción dinámico $\varphi^{\prime},{ }^{\circ}$ & 8 \\
\hline
\end{tabular}

Con base en los datos de la Tabla 2, se obtuvo una distancia de viaje de aproximadamente $65 \mathrm{~m}$. En la Figura 14 se presenta la superficie del talud cada $2 \mathrm{~s}$, durante los $40 \mathrm{~s}$ que tardó en detenerse la masa deslizada.

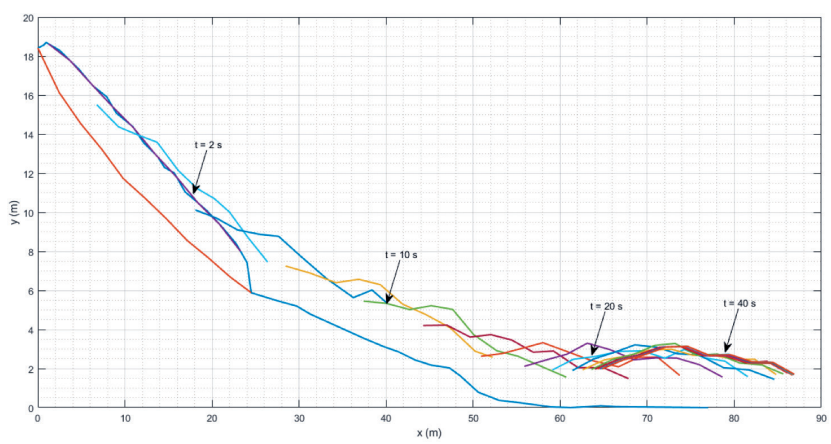

Figura 14: Superficie del talud durante el deslizamiento de Hiegaesi

Adicionalmente, de la Tabla 2 se obtuvieron las gráficas de posición y velocidad mostradas en la Figura 15, observándose que el deslizamiento tiende a mantener su posición y disminuir su velocidad significativamente alrededor de los $25 \mathrm{~s}$, evidenciándose que llega una distancia de viaje de aproximadamente $65 \mathrm{~m}$. La velocidad máxima fue alcanzada a $\operatorname{los} 4 \mathrm{~s}$, luego empieza a disminuir 
significativamente hasta que disipa completamente su energía cinética. La velocidad promedio durante el trayecto de la masa deslizada es de $1.62 \mathrm{~m} / \mathrm{s}$, clasificándose como un deslizamiento extremadamente rápido según Hungr et al. (2013). Dado que los valores de los parámetros para este deslizamiento fueron determinados mediante retro cálculo, se realizó un análisis de sensibilidad para evidenciar la variación en el resultado.

a)

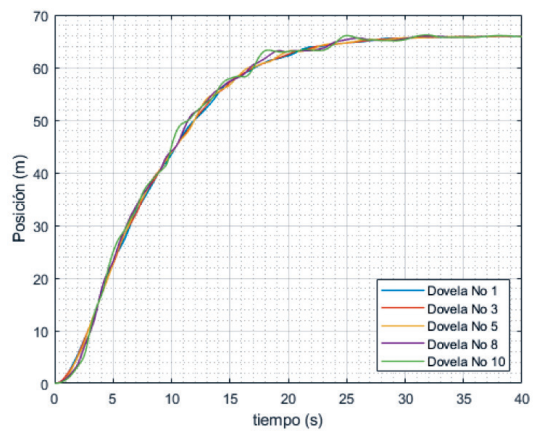

b)

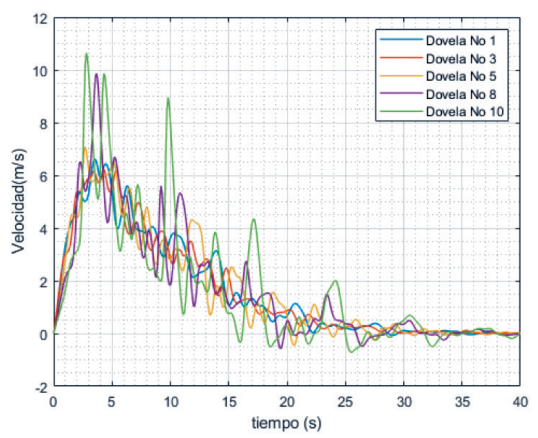

Figura 15: a) Posición y b) velocidad de cada dovela durante el deslizamiento Hiegaesi

a)
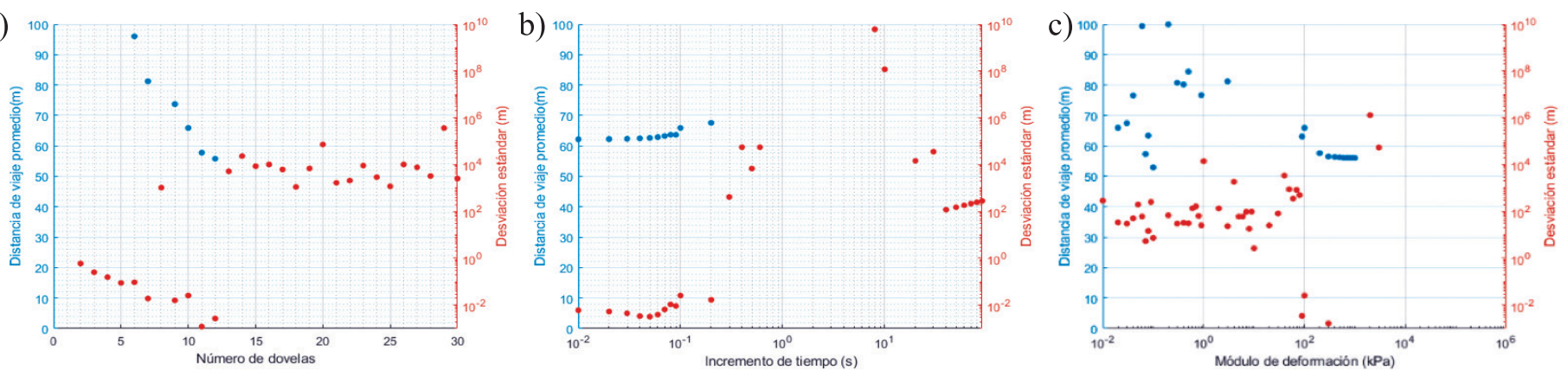

d)
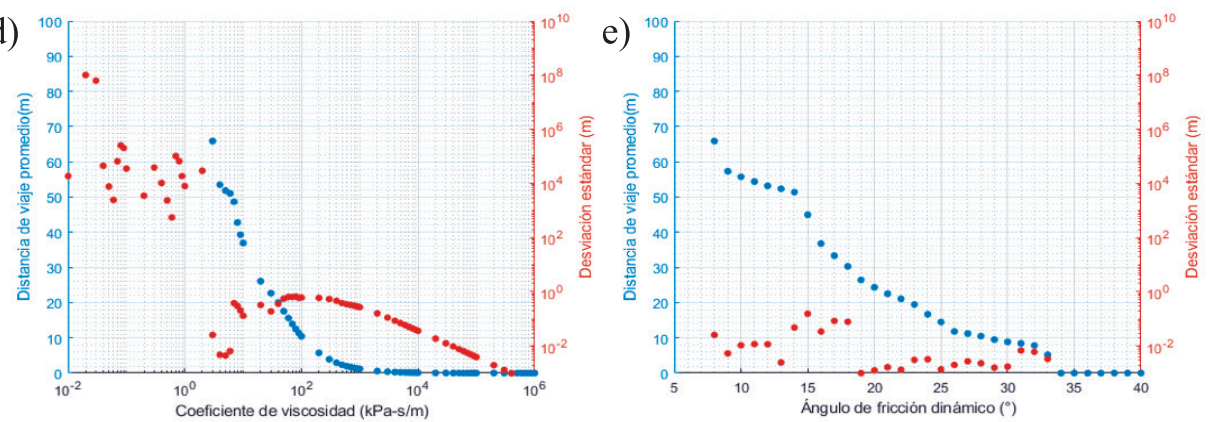

Figura 16: Análisis de sensibilidad para: a) número de dovelas, b) incremento de tiempo, c) módulo de deformación, d) coeficiente de viscosidad, e) ángulo de fricción dinámico
Como resultado, se puede observar en la Figura 16 que el máximo número de dovelas en el cual se puede discretizar la masa deslizada es 13 , debido principalmente a la irregularidad de la superficie de falla, lo cual conlleva a que varíe la pendiente por la cual viaja cada una de las dovelas, y así mismo, varíe el tamaño de las dovelas. El incremento de tiempo únicamente influye en la estabilidad del modelo. Para el caso de $E$, se encontró que no tiene influencia en la distancia de viaje, pero sí la tiene en la estabilidad del modelo (obteniendo un rango entre $100 \mathrm{y}$ $1000 \mathrm{kPa}$ ), debido a la irregularidad de tamaño en la masa deslizada, donde la diferencia de tamaño de cada una de las dovelas tiene influencia en los resultados obtenidos.

Se observa que la influencia de $\mu$ es muy fuerte en el resultado de distancia de viaje del deslizamiento. Finalmente, en el análisis de sensibilidad para $\varphi^{\prime}$, se observan cambios bruscos en los resultados de la distancia de viaje, lo cual es debido a los cambios de pendiente de la superficie que recorre la masa deslizada. No obstante, este parámetro influye únicamente, mediante una relación inversa, en la distancia de viaje.

Adicionalmente, se realizó el mismo procedimiento variando dos parámetros simultáneamente, con el fin de identificar que influencia tienen los parámetros en los resultados de distancia de viaje, en donde los puntos azules representan estabilidad del modelo, y los puntos amarillos 
a)

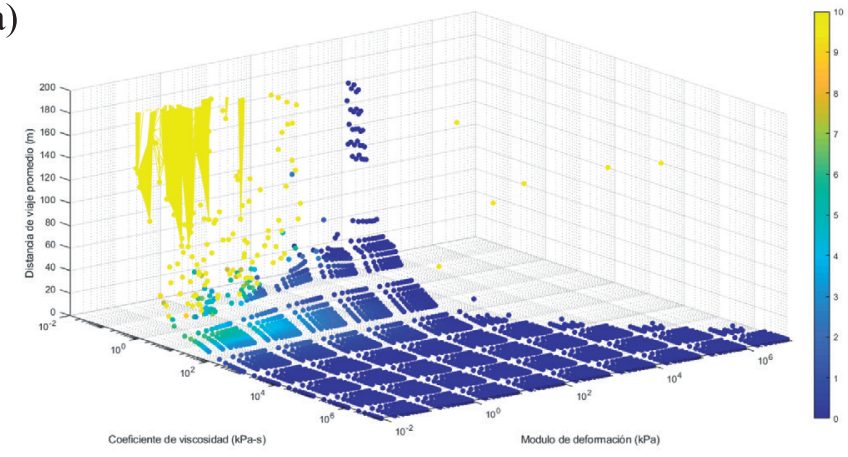

b)

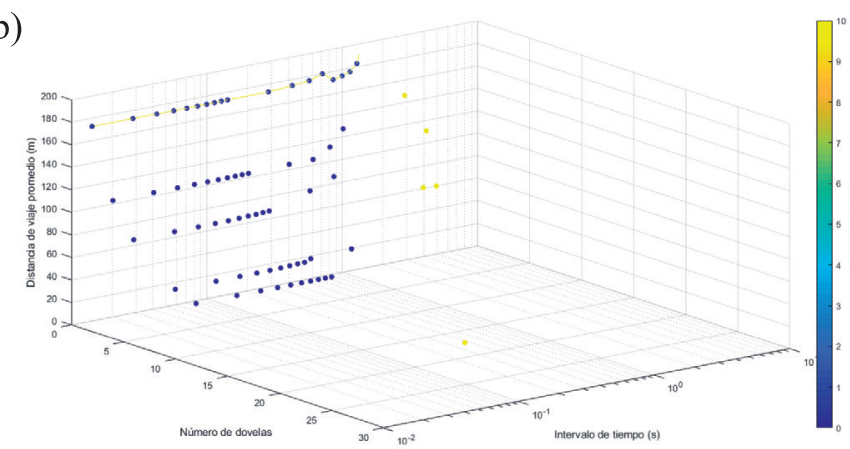

Figura 17: Análisis de sensibilidad para: a) módulo de deformación y coeficiente de viscosidad y b) incremento de tiempo y número de dovelas

representan inestabilidad numérica del modelo (véase Figura 17).

En la Figura 17a se observa que para $E>10^{3} \mathrm{kPa}$, debe existir una relación proporcional con $\mu$ para obtener resultados válidos; de lo contrario, el modelo se vuelve inestable numéricamente. Adicionalmente, en la Figura $17 \mathrm{~b}$ se observa que para discretizar más el espacio (mayor número de dovelas) es necesario discretizar más el tiempo (menor incremento de tiempo); lo cual es consecuente con los criterios de estabilidad para el método de diferencias finitas.

\section{Discusión de los resultados}

Entre las suposiciones ya mencionadas, se encuentra que al ser bloques conectados por resortes estos no se separan entre sí y mantienen el volumen constante durante el trayecto. Estas suposiciones hacen que el modelo sea una simplificación, respecto al alto grado de complejidad que puede llegar a tener el movimiento de los deslizamientos, donde en realidad es cada partícula de suelo la que interactúa con su alrededor, gobernada incluso por fuerzas no gravitatorias (viscosas y eléctricas) que conllevan al desplazamiento y rotación de cada una de estas. En el caso aplicado se evidencia en el reporte de Wang et al. (2002), que, por el efecto topográfico tridimensional, la masa deslizada se separó durante su trayecto y, por tanto, cambió su dirección. Sin embargo, al ser planteado como un problema de deformación plana, no es posible tener en cuenta estos efectos en el modelo propuesto. En este caso se debe escoger una sección (bidimensional) representativa de la ladera. A pesar de que en el caso en el que se aplicó el modelo, se logró simular el evento con resultados precisos, se evidenció a partir del análisis de sensibilidad realizado que en cada caso solo se tiene estabilidad numérica bajo ciertos rangos de parámetros. Además, se observó que existe inestabilidad numérica en ciertas dovelas, lo cual es reflejado en el comportamiento vibratorio en los valores obtenidos de velocidad. Los criterios de estabilidad y convergencia numérica conllevan a que se requiere que exista cierta relación entre los parámetros de la ecuación $(E, \mu$, masa de cada dovela y $\Delta t$ ) que eviten que los errores de truncación y/o redondeo se propaguen en cada iteración. Sin embargo, dada la complejidad de la ecuación de movimiento desarrollada para esta metodología, no fue posible obtener una expresión que garantice la estabilidad numérica, empero, se conoce que esta depende de los parámetros mencionados previamente. Como ya se ha indicado, $E$ corresponde más a un parámetro de ajuste numérico, debido a su influencia en los modelos ejecutados. Por otro lado, $\mu$ tiene gran influencia en los resultados de distancia de viaje; sin embargo, $\mu$ puede variar en órdenes de magnitud según los niveles de deformación en los que se realicen los ensayos. Esto implica que para su determinación se requiera más investigación para la condición dinámica de la masa deslizada.

Aunque se verificó que al aplicar la metodología en deslizamientos rápidos en suelos, es factible obtener resultados de distancia de viaje y velocidades de manera precisa, se resalta que estos resultados se obtuvieron retro calculando los parámetros. Así que para implementar la metodología en predicciones de distancia de viaje de deslizamientos es necesario estudiar más a fondo la estabilidad numérica del modelo planteado, y continuar investigando acerca de las fuerzas viscosas que actúan en este tipo de deslizamientos. 


\section{Conclusiones}

Se desarrolló un modelo que permite estimar la distancia de viaje y velocidad de deslizamientos en suelos, en los cuales se tiene en cuenta las variables geométricas, mecánicas y reológicas del deslizamiento. La metodología parte de las fuerzas resultantes del método de equilibrio límite y luego, el movimiento es modelado con un sistema de bloques conectados por resortes, en los que actúan fuerzas resistentes friccionales y viscosas. La ecuación de movimiento fue solucionada por el método de diferencias finitas. Debido a que la solución de la ecuación diferencial requiere de condiciones iniciales, fue necesario dividir el movimiento en dos etapas. La primera consiste en evaluar por medio de un método de equilibrio límite la estabilidad del talud que discretice el talud en dovelas y considere fuerzas actuantes entre ellas. Con las fuerzas resultantes entre dovelas, se planteó el procedimiento para calcular el primer desplazamiento de la masa deslizada. Las coordenadas iniciales del talud y las del primer movimiento, corresponden a las condiciones iniciales para dar solución a la ecuación de movimiento planteada (segunda etapa). Adicionalmente, para los casos modelados se realizó un análisis de sensibilidad de los parámetros establecidos en la ecuación de movimiento, en los que se demostró que la sensibilidad es afectada por el volumen de masa deslizada (tamaño de las dovelas) y por la geometría de la superficie que recorre la masa deslizada. Con el análisis realizado, se estableció para cada modelo el rango de parámetros bajo los cuales la solución es estable y/o representa de manera adecuada la naturaleza física del deslizamiento.

Debido a la fuerte sensibilidad en los parámetros en los resultados del modelo, se recomienda continuar trabajando en la investigación de los parámetros, específicamente en el módulo de deformación y el coeficiente de viscosidad teniendo en cuenta los niveles de deformación que se pueden presentar, antes de implementarlo en la predicción de la cinemática de los deslizamientos. Para dar un mejor entendimiento al modelo propuesto, se recomienda modelar los deslizamientos con los que se cuente suficiente información de caracterización geotécnica y reportes de distancia de viaje, y así seguir validando la aplicabilidad del modelo con diferentes casos.

\section{Referencias}

Angeli, M.G., Gasparetto, P., Menotti, R.M., Pasuto, A. and Silvano, S. (1996). A visco-plastic model for slope analysis applied to a mudslide in Cortina d'Ampezzo, Italy. Quarterly Journal of Engineering Geology and Hydrogeology 29(3), 233-240

Banco Mundial (2012). Análisis de la gestión del riesgo de desastres en Colombia. Un aporte para la construcción de políticas públicas. Vol. 1. Bogotá, Colombia

Chen, H. and Lee, C.F. (2000). Numerical simulation of debris flows. Canadian Geotechnical Journal 37(1), 146-160

Chen, H. and Lee, C.F. (2002). Runout analysis of slurry flows with Bingham model. Journal of Geotechnical and Geoenvironmental Engineering 128(12), 1032-1042

Corominas, J., Moya, J., Ledesma, A., Lloret, A. and Gili, J.A. (2005). Prediction of ground displacements and velocities from groundwater level changes at the Vallcebre landslide (Eastern Pyrenees, Spain). Landslides 2, 83-96

Crosta, G.B., Imposimato, S. and Roddeman, D.G. (2003). Numerical modelling of large landslides stability and runout. Natural Hazards and Earth System Science 3(6), 523-538

Cuomo, S. (2014). New advances and challenges for numerical modeling of landslides of the flow type. Procedia Earth and Planetary Science 9, 91-100

GB50021 (1995). Code for investigation of geotechnical engineering. National Standard of PRC. China Architecture \& Building Press, Beijing, China (in Chinese)

Glastonbury, J., Fell, R. and Mostyn, G.R. (2002). Report on the post-collapse behaviour of debris from rock slope failures. University of New South Wales, Sydney, Australia

Hungr, O. (1995). A model for the runout analysis of rapid flow slides, debris flows, and avalanches. Canadian Geotechnical Journal 32(4), 610-623

Hungr, O. (2009). Numerical modelling of the motion of rapid flow-like landslides for hazard assesment. KSCE Journal of Civil Engineering, 13(4), 281-287

Hungr, O., Leroueil, S. and Picarelli, L. (2014). The Varnes classification of landslide types, an update. Landslides 11, 167-194

Hunter, G. and Fell, R. (2003). Travel distance angle for "rapid" landslides in constructed and natural soil slopes. Canadian Geotechnical Journal 40(6), 1123-1141 
Johnson, A.M. (1970). Physical processes in geology: a method for interpretation of natural phenomena; intrusions in igneous rocks, fractures, and folds, flow of debris and ice. Freeman, Cooper \& Company

Keaton, J.K., Wartman, J., Anderson, S., Benoit, J., deLaChapelle, J., Gilbert, R. and Montgomery, D.R. (2014). The 22 March 2014 Oso Landslide, Snohomish County, Washington. Geotechnical Extreme Events Reconnaissance Association GEER-36, USA

McDougall, S. (2017). Landslide runout analysis - current practice and challenges. Canadian Geotechnical Journal 54(5), 605-620

Miao, T., Liu, Z., Niu, Y. and Ma, C. (2001). A sliding block model for the runout prediction of high-speed landslides. Canadian Geotechnical Journal 38(2), 217-226

Mora, C. (2011). Contención de taludes en estado viscoplástico. Tesis de maestría, Universidad Nacional de Colombia, Bogotá D.C., Colombia
Petley, D. (2012). Global patterns of loss of life from landslides. Geology 40(10), 927-930

van Asch, Th.W.J., van Beek, L.P.H. and Bogaard, T.A. (2007). Problems in predicting the mobility of slow-moving landslides.

Engineering Geology 91, 46-55

Wang, F.W., Sassa, K. and Wang, G. (2002). Mechanism of a long-runout landslide triggered by the August 1998 heavy rainfall in Fukushima Prefecture, Japan. Engineering Geology 63, 169-185

Wang, X. (2008). Geotechnical analysis of flow slides, debris flows, and related phenomena. $\mathrm{PhD}$ thesis, University of Alberta, Canada 